\title{
IMPLEMENTATION OF CRYPTOGRAPHIC ALGORITHM ON IOT DEVICE'S ID
}

\author{
Aditya Kurniawan,S.T. ", Ratna Mayasari, M.T., Dr. M. Ary \\ Murti,S.T,M.T.a \\ ${ }^{a}$ Telkom University, School of Electrical Engineering \\ Bandung, Indonesia
}

\begin{abstract}
Data security is one of many problems that we face in IoT implementation. One of the problems with data security is we cannot assure that the data we received in the server-side is truly our data that we send from client-side or sensor-side. With that problems, comes some solutions like encryption and decryption method or some basic authentication planted on the server-side. In this research, we propose some encryption and decryption method that can be easily implemented on both server and sensor-side and then we analyze the complexity of its algorithm. This research aims to create some authentication process for the data that server-side receive from the sensor-side by creating encrypted ID consisting of 6 digits of alphanumeric and useful for identification process in system, so the server can recognize the data and validate it.
\end{abstract}

Keywords-Network Security, Internet of Things, Algorithm Complexity, EncryptionDecryption

\section{INTRODUCTION}

Internet of things is an emerging topic of so many aspects in our life such as technical, social, and also economic [1]. It has so many positive effects for our daily routine. With the continuous development and application of the internet of things, its own hidden security problems increasingly appear [2]. Because of that, a new paradigm of data trust, security and privacy is required to face these future issues in the internet of things [3]. Until now, there are some way to secure our data traffic throughout internet of things based network by using some conventional encryption-decryption algorithm such as AES or IDEA [4]. However, due to limited resources of sensor-side device, the implementation of data security on it facing some challenges [5]. Besides that, mostly those secure algorithm are implemented on the server-side which means there is a chance that intruders could use the data because there is no implemented algorithm in the sensor-side device. Basically, we cannot create a perfect security for a system because the threat will always develop faster than security does. The security was built to prevent the thre at, that is the reality now adays.

On the other hand, most of IoT based devices have limited computing levels. So, implementing some security improvement will be another challenge. There are several method that will solve this problem like encryption using hashes [6], compressive sensing [7], or blockchain based [8]-[11]. But both require next level of programming and much more difficult to implement on the majority of IoT devices. This paper proposes much simpler algorithm to implement by using mathematical approach to encrypt and decrypt the device ID. The algorithm ensures that every time the device sends the data, it will get new ID.

\section{Proposed Algorithm}

In this research, we propose a new encryption algorithm to encrypt the data that sensorside send to the server-side. If the encryption process located on sensor-side device, then the decryption process is located on the server-side. This algorithm uses some mathematical operations and an XOR method. The main goal of this algorithm is not preventing an intruder from using our data but the goal is an intruder cannot recognize which sensor-side 
data he has been using. So basically this algorithm will make an intruder more difficult in order to creating a replicated data from some specific sensor-side device.

In our algorithm, there is an item created besides the ID, such as op. Op has a function to check and verify the encryption process whether it is success ful or not. Those two items are inserted into HTTP packet (in header) with json format and sent by sensor side devices. This header will be checked and verified in server-side. The results of this process will determine the server treatment for the corresponding packet. For exact composition of a HTTP header of a packet described in the Table 1.

Table 1 HTTP Header Composition

\begin{tabular}{ll}
\hline Parameter & Value \\
\hline application/type & application/json \\
\hline op & int (results from calculation process) \\
\hline ID & encrypted ID \\
\hline
\end{tabular}

The following lines are explanations about our algorithm in each server and sensor-side and the general process of the system proposed.

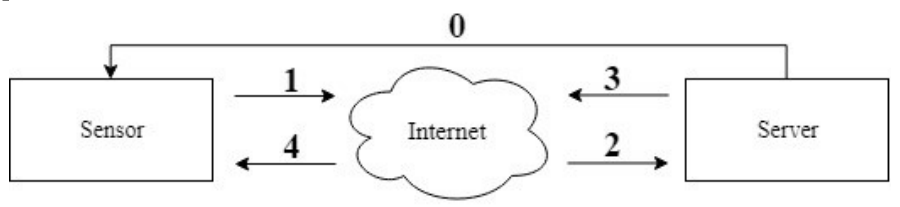

Figure 1 General process of the system

Our system consists of 2 main sections, sensor-side and server-side, both are connected via the internet connection. Communication process between them first occurs when server register sensor-side device in registration process. This process is performed by user through web application. Then the next step is to implement the ID that has been created before into the sensor-side devices. After that, the devices will start the sensing process and immediately sends the sensing data to the internet. But before it sends the data, it will encrypt the ID as an identification to the server. After the server has received the data, it will do the decryption process of the ID. If it was confirmed that the ID that received is correct, server will input the data to database and it will respond back to the sen sor-side by sending the sensor status. An explanation about those process above will be explained below.

\section{A. Server Side}

1) Creating the ID's

First of all, we create a string type ID that consist of alphabetical element without number and special character. Created ID's will be iterated into some numbers per alphabetical element. For this experiment system, we limit the maximum element in ID to a maximum of only 6 characters. The iteration starts from 0 to 25 where 0 equals to A, 1 equals to B, etc. (see Table 2 below). After that, we got a series of numbers that represents the alphabetical ID. These numbers will be implemented in the sensor-side device as their ID. Then the server computes some random series of 6 digits number known as unique ID and store both the ID and unique ID into the database. Some simple example listed below.

$\begin{array}{lccccccc}\text { Created ID } & : & \mathrm{C} & \mathrm{R} & \mathrm{Y} & \mathrm{P} & \mathrm{T} & \mathrm{O} \\ \text { Iterates } & : & 2 & 17 & 24 & 15 & 19 & 14 \\ \text { Unique ID } & : & 0 & 1 & 2 & 3 & 4 & 5\end{array}$


Table 2 Iteration Rules

\begin{tabular}{|c|c|c|c|c|c|c|c|c|c|c|c|c|}
\hline $\mathrm{A}$ & $\mathrm{B}$ & $\mathrm{C}$ & $\mathrm{D}$ & $\mathrm{E}$ & $\mathrm{F}$ & $\mathrm{G}$ & $\mathrm{H}$ & $\mathrm{I}$ & $\mathrm{J}$ & $\mathrm{K}$ & $\mathrm{L}$ & $\mathrm{M}$ \\
\hline $\mathbf{0}$ & $\mathbf{1}$ & $\mathbf{2}$ & $\mathbf{3}$ & $\mathbf{4}$ & $\mathbf{5}$ & $\mathbf{6}$ & $\mathbf{7}$ & $\mathbf{8}$ & $\mathbf{9}$ & $\mathbf{1 0}$ & $\mathbf{1 1}$ & $\mathbf{1 2}$ \\
\hline $\mathrm{N}$ & $\mathrm{O}$ & $\mathrm{P}$ & $\mathrm{Q}$ & $\mathrm{R}$ & $\mathrm{S}$ & $\mathrm{T}$ & $\mathrm{U}$ & $\mathrm{V}$ & $\mathrm{W}$ & $\mathrm{X}$ & $\mathrm{Y}$ & $\mathrm{Z}$ \\
\hline $\mathbf{1 3}$ & $\mathbf{1 4}$ & $\mathbf{1 5}$ & $\mathbf{1 6}$ & $\mathbf{1 7}$ & $\mathbf{1 8}$ & $\mathbf{1 9}$ & $\mathbf{2 0}$ & $\mathbf{2 1}$ & $\mathbf{2 2}$ & $\mathbf{2 3}$ & $\mathbf{2 4}$ & $\mathbf{2 5}$ \\
\hline
\end{tabular}

2) Decrypting the ID's

This process only occurs when we received some data from the sensor-side. Suppose we received a series of ID such as 396678. Then the server will executes searching procedure in the database looking for some data which has the exact same number of digits as the received ID. If the process ends up nothing means the ID is not from the database and the received data will be rejected. Meanwhile when the process found similar length of data, then it will executes subtraction operations between received ID and the data that has been found in searching procedure. The result of this subtraction operations must be a series of binary number, otherwise the data will be rejected. The final step of this process is operating some XOR operations into the result of those subtraction and match it up with the op that we received from sensor device. Some simple example listed below.

\begin{tabular}{llllllll} 
op & 0 & & & & & & \\
Received ID & $:$ & 3 & 9 & 6 & 6 & 7 & 8 \\
Found ID & $:$ & 2 & 9 & 6 & 6 & 6 & 7 \\
\hline Subtraction & $:$ & 1 & 0 & 0 & 0 & 1 & 1 \\
XOR & $:$ & 1 & & & & & \\
Results & $:$ & OP $\neq$ XOR & & & \\
Final Decision & $:$ & Reject
\end{tabular}

As we seen above, the result of XOR operation did not match with the op we received before. Then the next step is to calculate another ID found. If there is no another ID, the final decision is to reject the data that we receive and if the results of the calculation matched with op, then the server will do the next process. This process aims to verify the ID that has been sent to the server. To understand the process more easily, take a look into the flowchart below. 


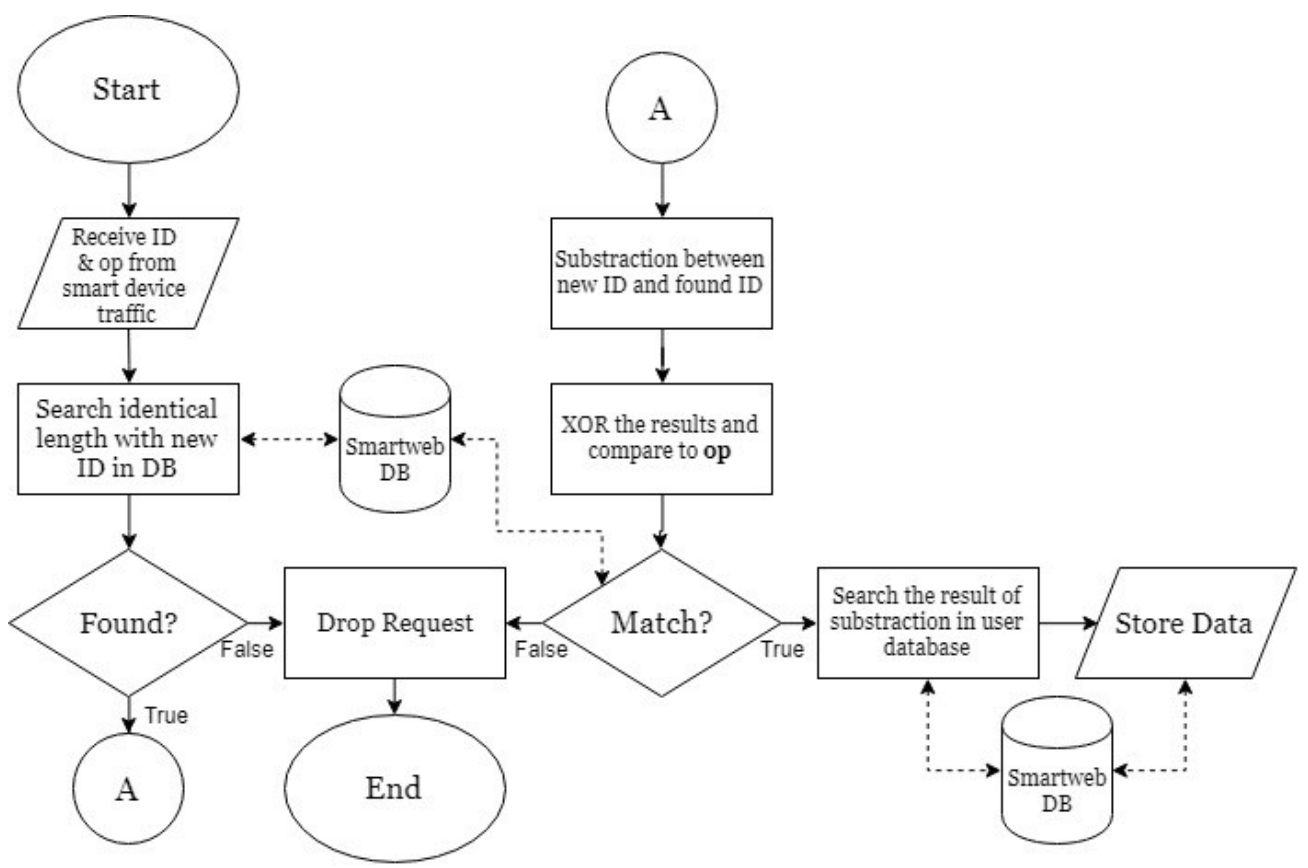

Figure 2 Decryption process flowchart

Suppose the calculation result is matched with op, then the next process is to search the corresponding user that has the device and store the data into their database.

\section{B. Sensor Side}

1) ID Iteration and Creating New ID's

Suppose we have implemented the ID into the device, the first job of sensor-side is to iterate the ID per alphabetical order and create some binary digits per iteration. For example, if we have 10 digits of ID, then the binary series will be 10 digits too. The next process is to perform some addition process of ID and binary series that resulting one new variable called new ID. This operation is only done when the results of addition is less than 10 otherwise it will automatically add the ID with 0 so the number will stay the same. A short example for the algorithm might be :

\begin{tabular}{llllllll} 
ID & $:$ & 3 & 9 & 5 & 6 & 7 & 8 \\
Binary & $:$ & 0 & 1 & 1 & 0 & 0 & 0 \\
\hline New ID & $:$ & 3 & 9 & 6 & 6 & 7 & 8
\end{tabular}$+$

After that, the device will operates some XOR operation into the series of binary resulting one final digits called op. This op will be sent also to the server together with the new ID. This process repeats itself when the device want to send data to the server. So every time the device sends the data, it gets brand new ID and op. Below is the flowchart of this process. 

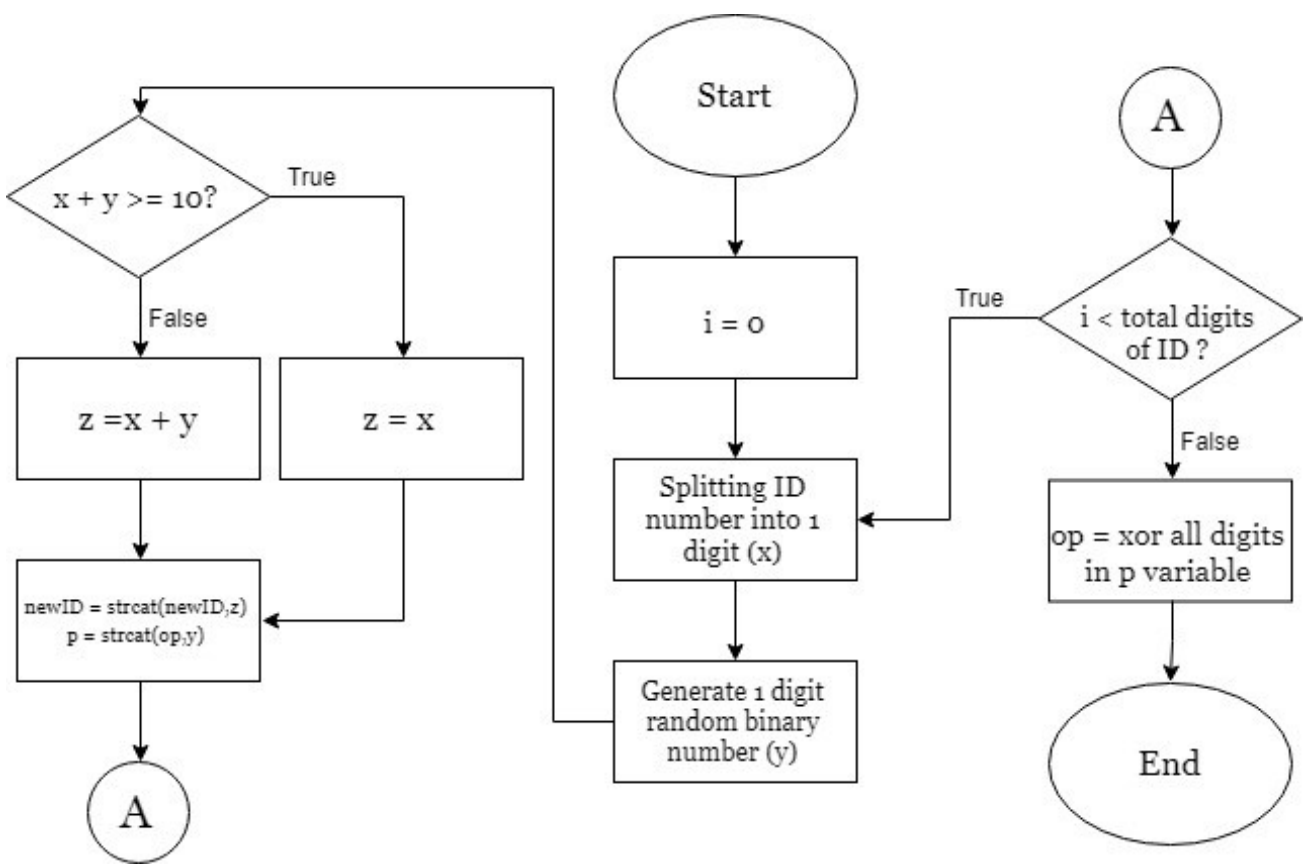

Figure 3 Encryption process flowchart

\section{IMPLEMENTATION AND RESULTS}

In the implementation, we used 6 bytes of text consisting of alphabet and number that will be encrypted through the process above and of course it can be more than 6 bytes of text that the system could encrypt. This system based on the research that has be en done by Nanda et al. [12]. In order to implement this algorithm on a large scale, we need to analyze the time complexity. The time complexity of an algorithm quantifies the amount of time taken by an algorithm to run as a function of the length of the string representing the input [13]. There are several ways to calculate the complexity of an algorithm such as complexity metrics [14] or using the big $\mathrm{O}$ notation. We used big O notation, where it represents the algorithm based on the input length. The result of this analysis could be the reason why we need this algorithm to be implemented on a large scale system.

\section{A. Device and Experiment Environment}

In this experiment, we use 2 device system called server side and sensor side. Each of them will be explained in the following lines.

\section{1) Server side}

In server-side section, we use regular server computer that used some HTTP server application such as Apache or NGINX. Our web applications use Laravel as PHP Framework. Laravel plays a role in data decryption process which implemented in their controller section (they use MVC model architecture).

2) Sensor side

Sensor-side system consist of 2 devices. First one is the sensor itself that have functions for sensing the data. Then another one is a single-board microcontroller (SBM) unit. In this experiment, we use ESP8266 model ESP-12 Node MCU v9. This SBM connects to the internet through IEEE $802.11 \mathrm{~b} / \mathrm{g} / \mathrm{n}$ connections.

\section{B. Calculation Results}

Then we calculate the big $\mathrm{O}$ notation of the encryption algorithm in the sensor-side device as shown in the section 2, we got the result as follows. 
Table 3 Encryption algorithm complexity

\begin{tabular}{ll}
\hline Parameter & Complexity \\
\hline Main Program & 1 \\
\hline $1^{\text {st }}$ for & $\mathrm{n}^{2}+9$ \\
\hline $2^{\text {nd }}$ for & $\mathrm{n}+1$ \\
\hline Complexity & $\mathrm{n}^{2}+\mathrm{n}+11$ \\
\hline Big O notation & $\mathrm{O}\left(\mathrm{n}^{2}\right)$ \\
\hline
\end{tabular}

And then for decryption process the result as follows.

Table 4 Decryption algorithm complexity

\begin{tabular}{ll}
\hline Parameter & Complexity \\
\hline $1^{\text {st }}$ for & $\mathrm{n}^{4}+28$ \\
\hline $2^{\text {nd }}$ for & $\mathrm{n}^{2}+7$ \\
\hline Check Index & $\mathrm{n}+5$ \\
\hline Complexity & $\mathrm{n}^{4}+\mathrm{n}^{2}+\mathrm{n}+40$ \\
\hline Big O notation & $\mathrm{O}\left(\mathrm{n}^{4}\right)$ \\
\hline
\end{tabular}

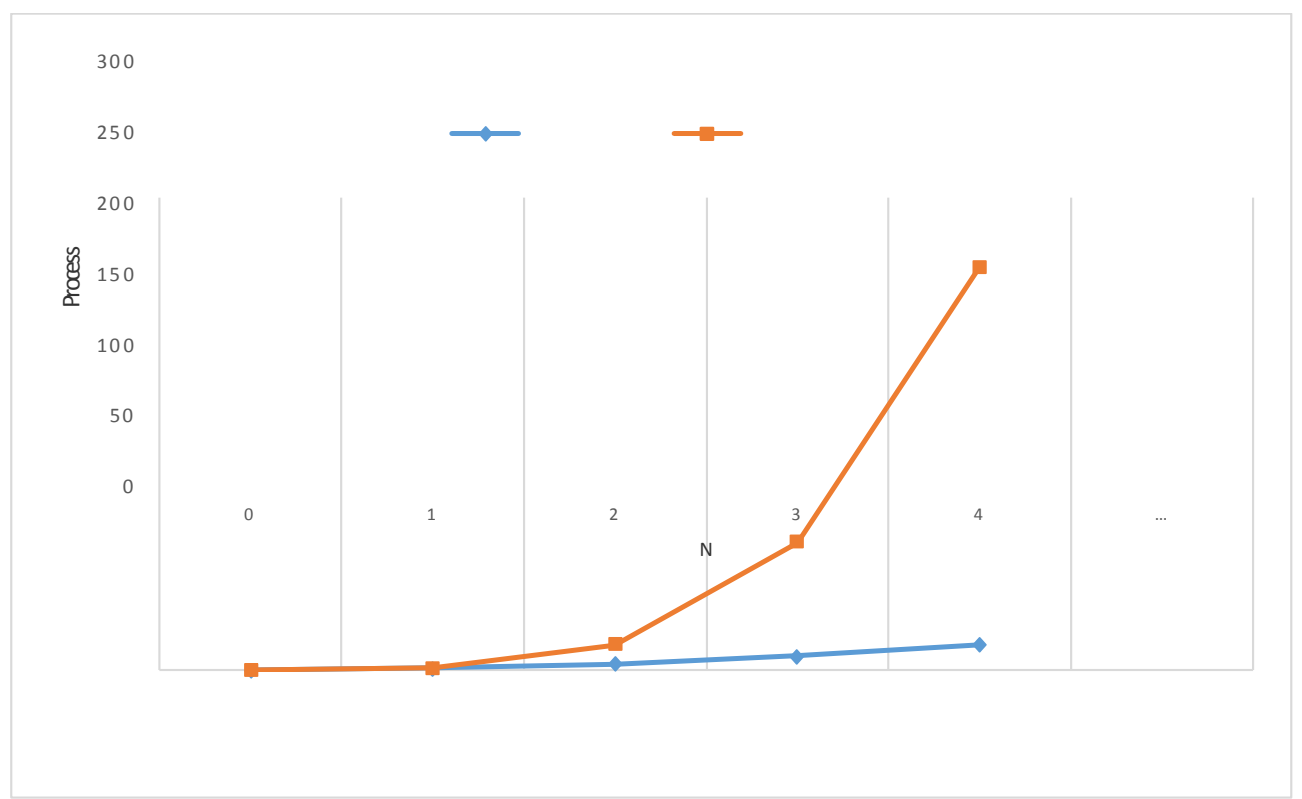

Figure 2 Big O notation graph

In the table 1 and 2 , it presents the complexity of encryption and decryption process. It shows that the encryption process big $\mathrm{O}$ notation is $\mathrm{O}(\mathrm{n} 2)$ while the decryption is $\mathrm{O}(\mathrm{n} 4)$. In figure 4, we see the comparison between those two process doing exactly the same amount of data processed $(\mathrm{N})$. The value of $\mathrm{N}$ will increase as many as the data processed throughout the system. We can see that the decryption process needs more time (process) to be done rather than the encryption process. This because the encryption process occurs on the sensor-side device which has small computation ability. On the other side, the decryption process occurs in the server side which has the best computational ability over the system. For those reason, we implement some algorithm on the sensor-side device simpler than the server.

\section{Conclusion}

As a conclusion of this research, the security part in IoT networks is highly needed. That is because nowadays, IoT networks almost reach every single thing that we used in 
everyday activities. Those device were simultaneously sends and receives sensitive data throughout the internet. Because of that, we need a security system that not only has a good security part but also got an advantage point in the performance part. By calculating the algorithm complexity using big $\mathrm{O}$ notation, we can know how good or how bad an algorithm was. Our implementation results showed that the decryption process has more complex than the encryption. That is because several factor such as low computing ability on the sensor-side device and perform some function to access the database on the server side makes more command executed on the server side. In the end, the results above showing the worst case running time of an algorithm, because the big $\mathrm{O}$ notation describes the upper bound of an element [15]. So if we want to implement the algorithm in bigger environment with more sensor-side device connected to the server, we need some algorithm that has less complexity compared to this one because we cannot waste the resource on the server on doing some process that we do not want to do.

In our future work, we aim to develop new algorithm that has less complexity compared to this one in both aspect of encryption and decryption process. Also we wanted to develop some security parts of these algorithm more efficient and also aim to implement the system to a larger environment with IoT based networks.

\section{REFERENCES}

[1] K. Rose, S. Eldridge, and L. Chapin, "THE INTERNET OF THINGS: AN OVERVIEW. Understanding the Issues and Challenges of a More Connected World.," Internet Soc., no. October, p. 80, 2015.

[2] X. F. Wang, "Research on Security Issues of the Internet of Things," 4th Int. Conf. Mater. Sci. Inf. Technol. MSIT 2014 989-994, vol. 994, pp. 4261-4264, 2014.

[3] A. Riahi, Y. Challal, E. Natalizio, Z. Chtourou, and A. Bouabdallah, "A Systemic Approach for IoT Security," 2013 IEEE Int. Conf. Distrib. Comput. Sens. Syst., no. May, pp. 351-355, 2013.

[4] R. Yegireddi and R. K. Kumar, "A survey on conventional encryption algorithms of Cryptography," Proc. 2016 Int. Conf. ICT Business, Ind. Gov. ICTBIG 2016, 2017.

[5] J. P. Walters, Z. Liang, W. Shi, and V. Chaudhary, "Wireless sensor network security: A survey," Secur. Distrib. Grid, Mobile, Pervasive Comput., pp. 367-410, 2007.

[6] M. A. Khan and K. Salah, "IoT security: Review, blockchain solutions, and open challenges," Futur. Gener. Comput. Syst., vol. 82, pp. 395-411, 2018.

[7] W. Xue, C. Luo, G. Lan, R. Rana, W. Hu, and A. Seneviratne, "Kryptein: A Compressive-sensing-based Encryption Scheme for the Internet of Things," Proc. 16th ACM/IEEE Int. Conf. Inf. Process. Sens. Networks, pp. 169-180, 2017.

[8] M. Samaniego and R. Deters, "Blockchain as a Service for IoT," Proc. - 2016 IEEE Int. Conf. Internet Things; IEEE Green Comput. Commun. IEEE Cyber, Phys. Soc. Comput. IEEE Smart Data, iThings-GreenCom-CPSCom-Smart Data 2016, pp. 433-436, 2017.

[9] A. Bahga and V. K. Madisetti, "Blockchain Platform for Industrial Internet of Things," J. Softw. Eng. Appl., vol. 09, no. 10, pp. 533-546, 2016.

[10] S. Huh, S. Cho, and S. Kim, "Managing IoT devices using blockchain platform," Int. Conf. Adv. Commun. Technol. ICACT, pp. 464-467, 2017.

[11] J. Ellul and G. J. Pace, "AlkylVM: A Virtual Machine for Smart Contract Blockchain Connected Internet of Things," 2018 9th IFIP Int. Conf. New Technol. Mobil. Secur. NTMS 2018 - Proc., vol. 2018-Janua, pp. 1-4, 2018.

[12] N. C. Pratama, M. A. Murti, and R. Mayasari, "SISTEM AUTOKONEKSI PERANGKAT CERDAS YANG DITERAPKAN PADA SISTEM PENGAWASAN DAN KONTROL DENGAN MENGGUNAKAN INTERNET 
OF THINGS," J. Penelit. dan Pengemb. Telekomun. Kendali, Komputer, Elektr. dan Elektron., vol. 1, no. 2, 2016.

[13] R. Vaz, “Automated Big-O Analysis of Algorithms,” pp. 2-7, 2017.

[14] M. Kayam, M. Fuwa, H. Kunimune, M. Hashimoto, and D. K. Asano, “Assessing your algorithm: A program complexity metrics for basic algorithmic thinking education," ICCSE 2016 - 11th Int. Conf. Comput. Sci. Educ., no. Iccse, pp. 309$313,2016$.

[15] C. C. E. Leiserson, R. R. L. Rivest, C. Stein, and T. H. Cormen, Introduction to Algorithms, Third Edition, 3rd ed., vol. 7, no. 3. London: The MIT Press, 2009. 